\title{
淮北相山恢复演替群落优势树种叶片的生态解剖
}

\author{
史刚荣 汤 盈 张 铮 \\ (淮北煤炭师范学院生物系,安徽淮北 235000)
}

\begin{abstract}
摘 要 对淮北相山混交林 5 个优势种叶片的生态解剖学观察表明, 其叶片结构具有一定的旱生特征: 表皮具发 达的表皮毛或角质层, 全棶等面叶或具发达的栅栏组织,维管组织发达。牡荆 (Vitex negundo var. cannabifolia) 和酸 菄 (Ziziphus jujuba var. spinosa) 作为两个广布优势树种, 叶片结构表现出很大的可塑性: 1) 同一群落环境(混交林) 中, 叶片结构随着季节的变化表现出发育可塑性 ( 5 月初的叶片比 9 月中旬更具有阳生叶的特点);2)不同恢复演替 阶段的群落中, 叶片结构随着群落环境的变化表现出环境可塑性, 其变化趋势为: 灌草丛 (旱生/阳生)-灌丛 (旱生/ 阳生)_落叶疏林(中生/阳生)-人工侧柏 (Platycladus orientalis) 林(中生/阴生)。这种可塑性既是植物适应其异质生 境的一种重要机制, 同时又是不同群落环境的反映。非参数相关分析表明, 告荆和酸杳的叶片结构受多个生态因 子综合影响, 其中水分和风速是影响叶片结构的主导因子。叶片的上表皮角质层厚度、气孔器密度、栅栏组织厚 度、叶片厚度、木质部厚度、韧皮部厚度、维管束厚度等性状均与土壤含水量和空气相对湿度呈显著负相关, 与风速 呈显著正相关。
\end{abstract}

关键词 剥蚀残丘 恢复演替 优势树种 叶片 表型可塑性 生态解剖学

\section{LEAF ANATOMY OF DOMINANT PLANT SPECIES IN THE SUCCESSIONAL COMMUNITIES OF XIANGSHAN MOUTNAIN, HUAIBEI, CHINA}

\author{
SHI Gang-Rong TANG Ying and ZHANG Zheng \\ (Department of Biology, Huaibei Coal Industry Teachers College, Huaibei, Anhui 235000, China)
}

\begin{abstract}
The results of eco-anatomical observations on the leaf blades of 5 dominant species in a mixed forest of Xiangshan Mountain, Huaibei, China, indicated that these species share some xeromorphic characteristics such as a developed epidermal cuticle or hairs, total-palisade or flourishing palisade tissue, and developed vascular tissue in middle vein. Ziziphus jujuba var. spinosa and Vitex negundo var. cannabifolia, the two dominant species widely distributed throughout Xiangshan Mountain, show great plasticity in their lamina structure in two aspects: 1) Within the same community, the lamina structure shows temporal developmental plasticity with leaves collected at the beginning of May being more characteristic of sun plants as compared to those in mid September; 2) In different successional community, lamina structure are indicative of environmental plasticity. The shrub-herb communities tend to have xeromorphic/sun leaves, shrub communities have xeromorphic/sun leaves, the sparse mixed forest has mesophytic/sun leaves, and the Platycladus orientalis forest has mesophytic/shade leaves. These differences in leaf anatomy reflect both the ability for plants to adapt heterogeneous habitats as well as differences in the environment of the different communities. The results of non-parameter correlation analysis showed that the lamina structure was influenced by many environment factors, with soil water content, relative humidity and wind velocity being the principle factors. Lamina anatomical characteristics, such as cuticle thickness of the upper epidermis, stomatal density, thickness of the palisade tissue, leaf thickness, xylem thickness and bundle thickness of the middle vein, all were negatively correlated to soil water content and relative humidity and positively correlated to wind velocity.
\end{abstract}

Key words Leaf structure, Lamina structure, Leaf blades, Phenotypic plasticity, Ecological anatomy

植物生态解剖学是运用植物形态解剖学的观察 和实验方法, 对生长在不同生境中的植物或演替系 列中的优势种进行研究, 探讨其在外部形态和内部
显微结构上的动态变化规律及与功能相互变化的科 学(彭少麟等, 2002)。叶片作为植物体暴露在环境 中面积最大的器官, 受水分、温度、光照等环境因子 
的影响显著(王勋陵和王静, 1989; Mott et al ., 1982)。 主要表现为叶外部形态的变化、叶厚度的变化及叶 内部解剖结构的差异 (郭玉华等, 2004; 刘全宏等, 2001; 费松林等, 1999; Rôças et al., 1997; Jackson, 1967; Wylie，1951）。这种表型可塑性（Phenotypic plasticity）被认为是植物克服环境异质性的重要途 径, 高的可塑性常常与对环境具有高的潜在适应能 力相关 (Strauss-Debenedetti \& Bazzaz, 1991)。

目前, 关于叶片生态解剖学的研究主要集中在 两个方面: 一是不同生态因子对植物叶片解剖结构 的影响 (蔡永立和达良俊, 2002; 刘全宏等, 2001; 方 精云等, 2000; 费松林等, 1999; 蔡永立等, 1999; 贺金 生等, 1994; Rôças et al., 1997; Bongers \& Popma, 1990; Carpenter \& Smith, 1975); 二是特定生境下植物 叶片的生态解剖学机制的研究 (张兴和杨晓杰, 2003; 黄振英等, 1997; 周广泰等, 1992; 王为义, 1985; Fahn, 1964, 1986)。另外, 对热带、亚热带群落演替 系列中的优势种的生态解剖学研究也有零星研究 （彭少麟等, 2002; Strauss-Debenedetti \& Berlyn, 1994), 但对温带退化生态系统中群落恢复演替不同阶段优 势树种叶片的解剖生态学研究却未见报道。

本文以淮北平原典型的剥蚀残丘一一相山群落 恢复演替不同阶段的 5 个优势种, 酸菄 (Ziziphus jujuba var. spinosa)、牡荆( Vitex negundo var. cannabifolia)、柘树 (Cudrania tricuspuidata)、构树 (Broussonetia papyrifera) 和小叶鼠李 (Rhamnus parvifolia) 为研究对 象, 通过对同一生境下不同优势树种, 以及群落恢复 演替不同阶段中广布优势种(酸隶和牡荆)叶片结构 的比较, 探讨了不同优势树种对相山干旱环境的趋 同适应特征, 揭示了广布优势种叶片的解剖可塑性 及其与生态因子之间的关系, 并就其群落学意义进 行了讨论。

\section{1 自然概况}

相山地处安徽淮北北部, 系泰山经鲁南山地, 自 徐州向南蜿蜒延伸的余脉, 为海拔 $100 \sim 350 \mathrm{~m}$ 的石 灰岩质岛状剥蚀残丘。本地区属于暖温带半湿润季 风气候, 常年降水较少, 年平均降水量约 $800 \sim 900$ $\mathrm{mm}$ 左右; 年平均气温在 $14 \sim 17{ }^{\circ} \mathrm{C}$ 之间, 全年无霜期 $220 \mathrm{~d}$ 左右; 日照时数 $2300 \sim 2500 \mathrm{~h}$ 左右, 土壤为石 灰土及淋溶褐土。相山山地侵蚀溶蚀严重, 岩石裸 露, 蓄水能力极差。由于长期人为活动的影响, 原有 的暖温带落叶阔叶林已不复存在, 大部分山丘为次 生裸地,主要植被类型有禾本科植物为优势种的草
丛, 酸柊、牡荆为优势种的灌丛或灌草丛, 以及局部 发育良好的人工侧柏 (Platycladus orientalis) 林和混 交林, 自然植被稀疏。

\section{2 材料与方法}

\section{1 研究材料}

实验材料分别于 2004 年 5 月和 9 月采自相山 东南坡植被恢复过程中不同演替阶段的群落中, 即 1)人工侧柏林: 侧柏占绝对优势, 林下和林隙夹杂着 一些酸杳、牡荆; 2)侧柏、构树混交林: 侧柏的密度相 对较小, 构树、柘树、酸杳、告荆等阔叶树种占优势; 3)牡荆、金色狗尾草 (Setaria glauca) 灌草丛: 自然植 被的草本层以金色狗尾草等禾本科植物占优势, 灌 木层以牡荆、酸杰为优势种; 4)酸柊、牡荆灌从: 以酸 本、告荆、小叶鼠李等灌木占优势。各树种的取样情 况及群落生境条件的实地观测数据见表 1 。生境条 件的观测于 2004 年 3 月 18 日至 9 月 20 日进行, 每 月按上、中、下旬各选择 1 个晴天, 于 $6: 00 \sim 7: 00$ 、 10:00 12:00、15:00 16:00、19:00 20:00 用 TN2280 型四合一表和 TES-1335 型照度计分别测定群 落环境的风速、空气相对湿度、温度和光照强度, 仪 器放置高度为 $2 \mathrm{~m}$ 。取地面以下 $10 \sim 20 \mathrm{~cm}$ 的土样, 保湿带回实验室用烘干法测定土壤含水量。每一样 点每种植物随机选 5 个植株, 每个植株按不同方向 在树冠中部取表层成熟叶 4 片, 以消除方向不同而 引起的差异。新鲜叶片沿中脉两侧剪取长 $1 \mathrm{~cm}$, 宽 $0.5 \mathrm{~cm}$ 的小片, 置 FAA 固定液中固定和保存。

\section{2 实验方法}

叶片结构采取常规石蜡切片法制片, 切片厚度 为 $12 \mu \mathrm{m}$, 番红-固绿对染, 加拿大树胶封片。用目镜 测微尺在光镜下测量叶片厚度、上下表皮厚度、叶肉 组织 (包括㮽栏组织厚度和海绵组织) 厚度、中脉厚 度、中脉维管束厚度等指标。叶表面结构用指甲油 涂于叶片表面取印记, 制成临时装片。用目镜测微 尺在光镜下测量气孔密度、气孔器长度等指标。每 项指标测量 40 组数据。

\section{3 统计分析}

用 One-Way ANOVA 揭示异质生境对叶片解剖 特征的影响。若主效应显著, 用 $L S D$ 进行多重比 较, 确定差异的显著程度 $(p=0.05)$ 。叶片结构的可 塑性指数 (Plasticity index, PI) 参考 Ashton 等( 1998) 的计算公式:

$P I=1-x / X$

其中 $x$ 为各种群中的最小平均值, $X$ 为各种群 
表 1 相山不同群落中的取样情况及其生态条件

Table 1 Leaf sampled and ecological conditions in different communities in Xiangshan

\begin{tabular}{|c|c|c|c|c|c|c|c|c|c|c|c|c|c|}
\hline $\begin{array}{c}\text { 群落 } \\
\text { Communities }\end{array}$ & $\begin{array}{c}\text { 树种 } \\
\text { Species }\end{array}$ & $\begin{array}{l}\mathrm{PH} \\
(\mathrm{m})\end{array}$ & $\begin{array}{l}\text { SH } \\
(\mathrm{m})\end{array}$ & $\begin{array}{l}\mathrm{CH} \\
(\mathrm{m})\end{array}$ & $\begin{array}{l}\text { Alt } \\
(\mathrm{m})\end{array}$ & $\begin{array}{l}\text { Slo } \\
\left({ }^{\circ}\right)\end{array}$ & $\begin{array}{l}\mathrm{CD} \\
(\%)\end{array}$ & $\begin{array}{l}\text { MLI } \\
(\mathrm{lx})\end{array}$ & $\begin{array}{l}\text { SWC } \\
(\%)\end{array}$ & $\begin{array}{c}\mathrm{RH} \\
(\%)\end{array}$ & $\begin{array}{l}\text { MAT } \\
\left({ }^{\circ} \mathrm{C}\right)\end{array}$ & $\begin{array}{l}\text { DDT } \\
\left({ }^{\circ} \mathrm{C}\right)\end{array}$ & $\begin{array}{c}\text { WV } \\
\left(\mathrm{m} \cdot \mathrm{s}^{-1}\right)\end{array}$ \\
\hline $\begin{array}{l}\text { 人工侧柏林 } \\
\text { Platycladus orientalis forest }\end{array}$ & $\begin{array}{l}(1)^{2)} \\
(2)^{2)}\end{array}$ & $\begin{array}{l}1.2 \\
0.8\end{array}$ & $\begin{array}{l}0.8 \\
0.6\end{array}$ & 9.3 & 145 & 26 & 96 & 9700 & 24.2 & 93.0 & 19.4 & 6.5 & 0.25 \\
\hline $\begin{array}{l}\text { 侧柏、构树混交林 } \\
\text { Platycladus orientalis }+ \text { Broussonetia pa- } \\
\text { pyrifera mixed forest }\end{array}$ & $\begin{array}{l}\left(1^{1 / 2)}\right. \\
\text { (2) }^{1 / 2)} \\
\text { (3) }{ }^{1)} \\
\text { (4) }^{1)} \\
\text { (5) }^{1)}\end{array}$ & $\begin{array}{l}1.6 \\
1.3 \\
3.2 \\
2.8 \\
0.8\end{array}$ & $\begin{array}{l}1.2 \\
1.1 \\
2.4 \\
2.0 \\
0.6\end{array}$ & 8.7 & 170 & 33 & 47 & 68700 & 19.4 & 89.1 & 21.8 & 8.7 & 0.42 \\
\hline $\begin{array}{l}\text { 酸本、牡荆灌丛 } \\
\text { Vitex negundo var. cannabifolia }+ \text { Ziziphus } \\
\text { jujuba var. spinosa shrub }\end{array}$ & $\begin{array}{l}\left(1^{2)}\right. \\
(2)^{2)}\end{array}$ & $\begin{array}{l}2.2 \\
1.7\end{array}$ & $\begin{array}{l}1.6 \\
1.2\end{array}$ & 2.3 & 340 & 12 & 0 & 83100 & 16.1 & 84.4 & 23.5 & 12.0 & 1.65 \\
\hline $\begin{array}{l}\text { 牡荆、金色狗尾草灌草丛 } \\
\text { Vitex negundo var. cannabifolia + Setaria } \\
\text { glauca shrub-herb }\end{array}$ & $\begin{array}{l}(1)^{2)} \\
(2)^{2)}\end{array}$ & $\begin{array}{l}1.8 \\
1.4\end{array}$ & $\begin{array}{l}1.5 \\
1.2\end{array}$ & 1.8 & 235 & 52 & 0 & 82500 & 14.7 & 78.2 & 22.7 & 11.2 & 1.84 \\
\hline
\end{tabular}

PH: 植株高度 Plant height SH: 取样高度 Sampling height CH: 群落高度 Community height Alt: 海拔 Altitude Slo: 坡度 Slope CD: 郁闭度 Canopy density MLI: 日均光强 Mean light intensity SWC: 土壤含水量 Soil water content RH: 空气相对湿度 Relative humidity MAT: 日均气温 Mean air temperature DDT: 日均温差 Difference of daily temperature WV: 风速 Wind velocity (1): 酸束 Ziziphus jujuba var. spinosa (2): 生荆 Vitex negundo var. cannabifolia (3): 柘树 Cudrania tricuspuidata (4): 构树 Broussonetia papyrifera (5): 小叶鼠李 Rhamnus parvifolia 1): 初夏叶(5 月取 样) Early summer leaves (collected in May) 2): 中秋叶(9月取样) Middle autumn leaves (collected in September)

中的最大平均值。用 Spearman 非参数相关分析揭 示生态因子对叶片结构的影响。

\section{3 结果与分析}

3.1 同一生境下不同优势树种的解剖学特征

在混交林的 5 个优势树种中, 构树叶片为全栅 型, 小叶鼠李、柘树为异面叶; 酸束和牡荆叶片的栅 栏组织和海绵组织的分化程度在不同叶片中变异很 大, 有的叶片为全㮽型叶, 有的叶片为异面叶, 称之 为全㮽异面混合型。叶片厚度 $115.1 \sim 195.5 \mu \mathrm{m}$; 上、下表皮均为单层细胞, 构树和牡荆的表皮具发达 的表皮毛; 上表皮细胞排列紧密, 具发达的角质膜, 上表皮厚度 $10.82 \sim 25.57 \mu \mathrm{m}$, 上表皮角质膜厚度 $3.44 \sim 4.74 \mu \mathrm{m}$ 。下表皮细胞形态常不规则, 厚度 $5.52 \sim 13.99 \mu \mathrm{m}$, 角质膜厚度 $2.88 \sim 4.68 \mu \mathrm{m}$ 。构树 的上表皮气孔密度为 $296.3 \mathrm{~mm}^{-2}$, 其余 4 种植物上 表皮无气孔分布。下表皮气孔密度 483.1 844.6 $\mathrm{mm}^{-2}$ 。栅栏组织发达, 排列紧密, 厚度约为 48.96 $96.48 \mu \mathrm{m}$; 海绵组织不甚发达, 厚度为 $36.46 \sim 77.49$ $\mu \mathrm{m}$; 海绵组织栅栏组织厚度比为 $0 \sim 1.04$ 。

5 种植物叶片中脉及其输导组织都比较发达, 中脉平均厚度为 $135.98 \sim 1341.90 \mu \mathrm{m}$ 。木质部平 均厚度为 $29.34 \sim 296.90 \mu \mathrm{m}$, 韧皮部平均厚度为 $21.35 \sim 117.79 \mu \mathrm{m}$, 木质部韧皮部厚度比为 $1.44 \sim$ 2.84 , 维管束平均厚度为 $50.69 \sim 414.69 \mu \mathrm{m}$, 维管束 中脉厚度比为 $0.28 \sim 0.42$ 。

3.2 两个广布优势树种叶片的解剖可塑性及其与
环境因子间的关系

\subsection{1 生荆和酸疋叶片结构的季相变化}

由表 2 可以看出, 混交林中牡荆和酸苯初夏叶 (5 月初)和中秋叶 (9 月中旬) 在解剖性状上存在显 著差异, 表明两种植物叶片结构随着季节的变化表 现出一定的发育可塑性。初夏叶上表皮厚度、上表 皮角质层厚度、气孔密度、栅栏组织厚度、中脉木质 部厚度、中脉韧皮部厚度、木质部韧皮部厚度比、维 管束中脉厚度比等性状显著大于中秋叶, 表明初夏 叶比中秋叶更具有阳生叶的特点, 这与初夏群落较 小郁闭度以及低温、低湿等环境条件相适应。牡荆 初夏叶和中秋叶解剖性状的差异程度比酸疋大, 说 明其叶片更易受季节变化的影响。

\subsection{2 牡荆和酸菄叶片结构的空间变化}

从表 3 和表 4 可以看出, 牡荆和酸疋的叶片结 构在不同群落中表现较大的可塑性, 其中以叶表皮 性状(如上表皮角质层厚度、下表皮毛密度、气孔密 度) 和叶肉性状 (如栅栏组织厚度、海绵组织厚度、海 绵组织栅栏组织厚度比、叶厚等) 的可塑性最为显 著, 而中脉性状相对较小。在灌从和灌草丛群落中, 两个树种的叶片均表现出典型的旱生/阳生结构: 上 表皮角质层较厚, 下表皮密被表皮毛(牡荆)或覆盖 较厚的角质层, 叶片显著增厚, 棚栏组织发达, 部分 叶片特化成全栅型, 海绵组织极不发达, 这与其土层 较薄、水分短缺、光照强度大等生境条件是相适应 的。相反, 生长在侧柏林下的牡荆和酸苯在叶片结 构上却表现出典型的阴生特点: 上表皮细胞外向壁 
表 2 混交林 5 个优势树种叶片的解剖特征

Table 2 Anatomic traits of leaves of five dominant species in mixed forest

\begin{tabular}{|c|c|c|c|c|c|c|c|}
\hline & $\left(1^{1)}\right.$ & $\left(1^{2)}\right.$ & (2) ${ }^{1)}$ & $(2)^{2)}$ & (3) $)^{\text {) }}$ & (4) $)^{1)}$ & (5) ${ }^{1)}$ \\
\hline $\operatorname{TUE}(\mu \mathrm{m})$ & $25.57 \pm 3.48$ & $33.41 \pm 8.30^{* *}$ & $10.82 \pm 2.46$ & $13.10 \pm 2.80^{* *}$ & $14.50 \pm 3.36$ & $19.10 \pm 2.73$ & $14.46 \pm 1.87$ \\
\hline $\operatorname{CTUE}(\mu \mathrm{m})$ & $4.74 \pm 2.27$ & $3.80 \pm 1.35^{*}$ & $3.44 \pm 0.91$ & $2.34 \pm 0.77^{* *}$ & $4.16 \pm 1.40$ & $4.38 \pm 1.16$ & $4.37 \pm 1.08$ \\
\hline $\operatorname{SDUE}\left(\mathrm{mm}^{-2}\right)$ & 0 & 0 & 0 & 0 & 296.3 & 0 & 0 \\
\hline $\operatorname{TLE}(\mu \mathrm{m})$ & $13.99 \pm 4.16$ & $17.47 \pm 4.73^{* *}$ & $9.60 \pm 1.93$ & $9.74 \pm 2.13$ & $5.52 \pm 1.65$ & $13.39 \pm 2.89$ & $10.68 \pm 2.82$ \\
\hline $\operatorname{CTLE}(\mu \mathrm{m})$ & $4.38 \pm 1.81$ & $2.84 \pm 0.91^{* *}$ & $3.06 \pm 0.94$ & - & $2.88 \pm 0.73$ & $4.68 \pm 1.06$ & $4.19 \pm 1.09$ \\
\hline $\operatorname{SDLE}\left(\mathrm{mm}^{-2}\right)$ & 483.1 & 459.1 & 844.6 & 780.2 & 716.0 & 654.3 & 781.3 \\
\hline $\mathrm{TPT}(\mu \mathrm{m})$ & $86.09 \pm 25.88$ & $66.16 \pm 26.06^{* *}$ & $96.48 \pm 34.56$ & $72.99 \pm 24.10^{* *}$ & $87.51 \pm 12.41$ & $75.87 \pm 10.41$ & $48.96 \pm 9.82$ \\
\hline $\operatorname{TST}(\mu \mathrm{m})$ & $14.00 \pm 19.60$ & $38.00 \pm 17.10^{* *}$ & $33.59 \pm 30.97$ & $49.45 \pm 14.30^{* *}$ & 0 & $77.49 \pm 19.93$ & $36.46 \pm 9.09$ \\
\hline TST/TPT & $0.28 \pm 0.41$ & $0.68 \pm 0.37^{* *}$ & $0.50 \pm 0.50$ & $0.82 \pm 0.57^{* *}$ & $0.00 \pm 0.00$ & $1.04 \pm 0.31$ & $0.79 \pm 0.31$ \\
\hline $\operatorname{TL}(\mu \mathrm{m})$ & $149.4 \pm 15.2$ & $155.1 \pm 31.4$ & $155.9 \pm 18.4$ & $145.3 \pm 23.5^{*}$ & $115.1 \pm 15.7$ & $195.5 \pm 27.3$ & $118.9 \pm 13.2$ \\
\hline $\mathrm{XT}(\mu \mathrm{m})$ & $100.25 \pm 19.99$ & $80.22 \pm 21.32^{* *}$ & $140.45 \pm 30.52$ & $78.35 \pm 17.45^{* *}$ & $296.90 \pm 64.09$ & $174.80 \pm 37.22$ & $29.34 \pm 4.75$ \\
\hline $\mathrm{PT}(\mu \mathrm{m})$ & $62.48 \pm 14.00$ & $74.02 \pm 17.53^{*}$ & $57.04 \pm 14.81$ & $48.72 \pm 8.31^{*}$ & $117.79 \pm 21.51$ & $63.35 \pm 15.90$ & $21.35 \pm 6.00$ \\
\hline $\mathrm{XT} / \mathrm{PT}$ & $1.64 \pm 0.33$ & $1.10 \pm 0.24^{* *}$ & $2.51 \pm 0.75$ & $1.66 \pm 0.49^{* *}$ & $2.17 \pm 0.54$ & $2.84 \pm 0.60$ & $1.44 \pm 0.32$ \\
\hline $\mathrm{TB}(\mu \mathrm{m})$ & $162.72 \pm 30.99$ & $154.24 \pm 34.86$ & $197.49 \pm 33.37$ & $127.07 \pm 19.96^{* *}$ & $414.69 \pm 73.70$ & $238.14 \pm 48.70$ & $50.69 \pm 8.96$ \\
\hline $\operatorname{TMV}(\mu \mathrm{m})$ & $382.80 \pm 54.21$ & $426.38 \pm 82.82^{* *}$ & $692.90 \pm 109.50$ & $646.50 \pm 111.60$ & $1341.90 \pm 162.83$ & $599.14 \pm 177.72$ & $135.98 \pm 11.37$ \\
\hline TRBM & $0.42 \pm 0.04$ & $0.36 \pm 0.06^{* *}$ & $0.29 \pm 0.05$ & $0.20 \pm 0.03^{* *}$ & $0.31 \pm 0.05$ & $0.41 \pm 0.06$ & $0.37 \pm 0.06$ \\
\hline
\end{tabular}

TUE: 上表皮厚度 Thickness of upper epidermis CTUE: 上表皮角质层厚度 Cuticle thickness of upper epidermis SDUE: 上表皮气孔密度 Stoma density of upper epidermis TLE: 下表皮厚度 Thickness of lower epidermis CTLE: 下表皮角质层厚度 Cuticle thickness of lower epidermis SDLE: 下表皮 气孔密度 Stoma density of lower epidermis TPT: 栅栏组织厚度 Thickness of palisade tissue TST: 海绵组织厚度 Thickness of spongy tissue TL: 叶厚 Thickness of leaves XT: 木质部厚度 Xylem thickness PT: 韧皮部厚度 Phloem thickness TB: 维管束厚 Bundle thickness TMV: 中脉厚度 Thickness of middle vein TRBM: 维管束中脉厚度比 Thickness ratio of bundle and middle vein (1) (5)、1)、2): 见表 1 See Table $1 \quad * *: p<0.01 \quad *: p<0.05$

表 3 不同群落环境中牡荆叶片的解剖特征

Table 3 Leaf anatomic characters of Vitex negundo var. cannabifolia living in different communities

\begin{tabular}{|c|c|c|c|c|c|}
\hline & $\begin{array}{c}\text { 人工侧柏林 } \\
\text { Platycladus } \\
\text { orientalis forest }\end{array}$ & $\begin{array}{c}\text { 侧柏、构树混交林 } \\
\text { Platycladus orientalis + } \\
\text { Broussonetia papyrifera } \\
\text { mixed forest }\end{array}$ & $\begin{array}{c}\text { 酸杳、牡荆灌丛 } \\
\text { Vitex negundo var. } \\
\text { cannabifolia }+ \text { Ziziphus } \\
\text { jujuba var. spinosa shrub }\end{array}$ & $\begin{array}{l}\text { 牡荆、金色狗尾草灌草丛 } \\
\text { Vitex negundo var. } \\
\text { cannabifolia + Setaria } \\
\text { glauca } \text { shrub-herb }\end{array}$ & $\begin{array}{l}\text { 可塑性指数 } \\
\text { Plasticity index }\end{array}$ \\
\hline $\operatorname{CTUE}(\mu \mathrm{m})$ & $0.80 \pm 0.37^{\mathrm{A}}$ & $2.34 \pm 0.77^{\mathrm{B}}$ & $2.61 \pm 0.42^{\mathrm{B}}$ & $3.21 \pm 0.76^{\mathrm{C}}$ & 0.75 \\
\hline $\operatorname{TUE}(\mu \mathrm{m})$ & $13.14 \pm 3.44^{\mathrm{a}}$ & $13.14 \pm 2.78^{\mathrm{a}}$ & $13.20 \pm 2.30^{\mathrm{a}}$ & $14.22 \pm 2.78^{\mathrm{a}}$ & 0.08 \\
\hline $\operatorname{TLE}(\mu \mathrm{m})$ & $10.15 \pm 2.43^{\mathrm{a}}$ & $9.74 \pm 2.13^{\mathrm{a}}$ & $8.79 \pm 3.87^{\mathrm{a}}$ & $9.81 \pm 2.46^{\mathrm{a}}$ & 0.13 \\
\hline $\operatorname{HDLE}\left(\mathrm{mm}^{-1}\right)$ & $9.09 \pm 17.09^{A}$ & $64.02 \pm 20.44^{\mathrm{B}}$ & $56.06 \pm 21.48^{\mathrm{B}}$ & $41.67 \pm 19.93^{\mathrm{C}}$ & 0.86 \\
\hline $\operatorname{SDLE}\left(\mathrm{mm}^{-2}\right)$ & $707.8 \pm 254.6^{\mathrm{A}}$ & $780.2 \pm 240.7^{\mathrm{A}}$ & $975.7 \pm 309.5^{\mathrm{B}}$ & $994.8 \pm 266.2^{\mathrm{B}}$ & 0.30 \\
\hline $\operatorname{SL}(\mu \mathrm{m})$ & $19.32 \pm 2.05^{\mathrm{A}}$ & $22.10 \pm 2.69^{\mathrm{B}}$ & $21.60 \pm 2.08^{\mathrm{b}}$ & $20.21 \pm 2.83^{\mathrm{a}}$ & 0.13 \\
\hline $\operatorname{TPT}(\mu \mathrm{m})$ & $34.13 \pm 12.04^{\mathrm{A}}$ & $72.99 \pm 24.10^{\mathrm{B}}$ & $103.17 \pm 20.43^{\mathrm{C}}$ & $113.93 \pm 35.09^{\mathrm{C}}$ & 0.70 \\
\hline LPC & $1 \sim 2$ & $2 \sim 4$ & $3 \sim 5(7)$ & $3 \sim 5(7)$ & - \\
\hline $\operatorname{TST}(\mu \mathrm{m})$ & $38.88 \pm 9.95^{\mathrm{A}}$ & $49.45 \pm 14.31^{\mathrm{bc}}$ & $43.97 \pm 11.96^{\mathrm{ac}}$ & $52.82 \pm 20.60^{\mathrm{b}}$ & 0.26 \\
\hline TST/TPT & $1.23 \pm 0.41^{\mathrm{A}}$ & $0.82 \pm 0.57^{\mathrm{b}}$ & $0.44 \pm 0.13^{\mathrm{C}}$ & $0.59 \pm 0.30^{c}$ & 0.64 \\
\hline $\mathrm{TL}(\mu \mathrm{m})$ & $96.29 \pm 19.98^{\mathrm{A}}$ & $145.32 \pm 23.49^{\mathrm{B}}$ & $169.12 \pm 21.29^{c}$ & $180.77 \pm 28.11^{\mathrm{d}}$ & 0.47 \\
\hline $\mathrm{XT}(\mu \mathrm{m})$ & $72.50 \pm 20.53^{\mathrm{a}}$ & $78.35 \pm 17.45^{\mathrm{ab}}$ & $80.28 \pm 23.66^{\mathrm{b}}$ & $85.66 \pm 23.53^{\mathrm{ab}}$ & 0.15 \\
\hline $\mathrm{PT}(\mu \mathrm{m})$ & $45.54 \pm 11.37^{\mathrm{A}}$ & $48.72 \pm 8.31^{\mathrm{a}}$ & $56.85 \pm 14.44^{\mathrm{B}}$ & $58.82 \pm 16.59^{\mathrm{b}}$ & 0.23 \\
\hline $\mathrm{XT} / \mathrm{PT}$ & $1.63 \pm 0.43^{\mathrm{a}}$ & $1.66 \pm 0.49^{\mathrm{a}}$ & $1.47 \pm 0.46^{\mathrm{a}}$ & $1.49 \pm 0.34^{\mathrm{a}}$ & 0.11 \\
\hline $\mathrm{TB}(\mu \mathrm{m})$ & $118.04 \pm 28.44^{\mathrm{a}}$ & $127.07 \pm 19.96^{\mathrm{ab}}$ & $137.13 \pm 29.51^{\mathrm{C}}$ & $144.48 \pm 34.24^{\mathrm{bc}}$ & 0.18 \\
\hline $\operatorname{TMV}(\mu \mathrm{m})$ & $539.09 \pm 129.27^{\mathrm{A}}$ & $646.46 \pm 111.61^{\mathrm{b}}$ & $633.65 \pm 101.93^{b}$ & $671.88 \pm 135.79^{\mathrm{b}}$ & 0.20 \\
\hline TRBM & $0.22 \pm 0.03^{\mathrm{a}}$ & $0.20 \pm 0.03^{\mathrm{a}}$ & $0.22 \pm 0.03^{\mathrm{a}}$ & $0.22 \pm 0.04^{\mathrm{a}}$ & 0.09 \\
\hline
\end{tabular}

HDLE: 下表皮毛密度 Hair density of lower epidermis SL: 气孔器长度 Stoma length LPC: 栅栏细胞层数 Layer of palisade cell 其它见表 2 Others see Table 2 相同字母的数字之间无显著差异 $(p=0.05)$, 带大写字母的数字与带其它不同字母的数字之间的差异极显著 $(p<0.01)$ The numbers sharing the same letter $(\mathrm{s})$ are not significantly different $(p=0.05)$, and the numbers with capitals and these with other different letters are very significantly different $(p<0.01)$ 
表 4 不同群落环境中酸苯叶片的解剖特征

Table 4 Leaf anatomic characters of Ziziphus jujuba living in different communities

\begin{tabular}{|c|c|c|c|c|c|}
\hline & $\begin{array}{c}\text { 人工侧柏林 } \\
\text { Platycladus } \\
\text { orientalis forest }\end{array}$ & $\begin{array}{c}\text { 侧柏、构树混交林 } \\
\text { Platycladus orientalis + } \\
\text { Broussonetia papyrifera } \\
\text { mixed forest }\end{array}$ & $\begin{array}{c}\text { 酸柊、牡荆灌从 } \\
\text { Vitex negundo } \text { var. } \\
\text { cannabifolia }+ \text { Ziziphus } \\
\text { jujuba var. spinosa shrub }\end{array}$ & $\begin{array}{l}\text { 士荆、金色狗尾草灌草丛 } \\
\text { Vitex negundo var. } \\
\text { cannabifolia }+ \text { Setaria } \\
\text { glauca } \text { shrub-herb }\end{array}$ & $\begin{array}{l}\text { 可塑性指数 } \\
\text { Plasticity index }\end{array}$ \\
\hline $\operatorname{CTUE}(\mu \mathrm{m})$ & $1.38 \pm 0.73^{\mathrm{A}}$ & $3.80 \pm 1.35^{\mathrm{b}}$ & $4.00 \pm 0.50^{\mathrm{b}}$ & $4.03 \pm 0.84^{\mathrm{b}}$ & 0.66 \\
\hline $\operatorname{TUE}(\mu \mathrm{m})$ & $25.89 \pm 5.71^{\mathrm{A}}$ & $33.41 \pm 8.37^{\mathrm{b}}$ & $35.00 \pm 5.76^{\mathrm{b}}$ & $33.87 \pm 8.13^{\mathrm{b}}$ & 0.26 \\
\hline $\operatorname{TLE}(\mu \mathrm{m})$ & $16.64 \pm 8.00^{\mathrm{a}}$ & $17.47 \pm 4.73^{\mathrm{a}}$ & $15.80 \pm 3.92^{\mathrm{a}}$ & $19.96 \pm 14.05^{\mathrm{a}}$ & 0.21 \\
\hline $\operatorname{CTLE}(\mu \mathrm{m})$ & $0.97 \pm 0.30^{A}$ & $2.84 \pm 0.91^{\mathrm{B}}$ & $3.40 \pm 0.73^{\mathrm{D}}$ & $4.09 \pm 1.00^{\mathrm{C}}$ & 0.76 \\
\hline $\operatorname{SDLE}\left(\mathrm{mm}^{-2}\right)$ & $352.0 \pm 217.4^{\mathrm{a}}$ & $459.1 \pm 283.5^{\mathrm{c}}$ & $554.8 \pm 236.4^{\mathrm{B}}$ & $566.3 \pm 221.5^{\mathrm{B}}$ & 0.38 \\
\hline $\mathrm{SL}(\mu \mathrm{m})$ & $22.10 \pm 2.27^{\mathrm{A}}$ & $24.99 \pm 1.95^{\mathrm{b}}$ & $25.71 \pm 2.36^{\mathrm{b}}$ & $23.44 \pm 2.47^{\mathrm{A}}$ & 0.14 \\
\hline $\operatorname{TPT}(\mu \mathrm{m})$ & $25.58 \pm 8.23^{\mathrm{A}}$ & $66.16 \pm 26.06^{\mathrm{B}}$ & $84.35 \pm 25.89^{\mathrm{C}}$ & $108.29 \pm 44.86^{\mathrm{D}}$ & 0.76 \\
\hline LPC & $1(2)$ & $2 \sim 4$ & $3 \sim 5(7)$ & $3 \sim 5(7)$ & - \\
\hline $\operatorname{TST}(\mu \mathrm{m})$ & $34.29 \pm 10.24^{\mathrm{a}}$ & $38.03 \pm 17.11^{\mathrm{a}}$ & $40.58 \pm 24.15^{\mathrm{a}}$ & $27.20 \pm 28.31^{\mathrm{a}}$ & 0.33 \\
\hline TST/TPT & $1.40 \pm 0.42^{\mathrm{A}}$ & $0.68 \pm 0.37^{\mathrm{b}}$ & $0.58 \pm 0.38^{\mathrm{b}}$ & $0.40 \pm 0.42^{\mathrm{c}}$ & 0.71 \\
\hline $\operatorname{TL}(\mu \mathrm{m})$ & $103.25 \pm 17.57^{\mathrm{A}}$ & $155.06 \pm 31.43^{\mathrm{B}}$ & $175.73 \pm 16.01^{\mathrm{C}}$ & $184.97 \pm 32.99^{\mathrm{C}}$ & 0.44 \\
\hline $\mathrm{XT}(\mu \mathrm{m})$ & $66.44 \pm 35.93^{\mathrm{a}}$ & $80.22 \pm 21.32^{\mathrm{ab}}$ & $93.01 \pm 28.70^{\mathrm{bc}}$ & $101.27 \pm 20.32^{\mathrm{C}}$ & 0.34 \\
\hline $\mathrm{PT}(\mu \mathrm{m})$ & $66.67 \pm 18.22^{\mathrm{a}}$ & $74.02 \pm 17.53^{\mathrm{ab}}$ & $84.83 \pm 28.66^{\mathrm{B}}$ & $79.76 \pm 18.30^{\mathrm{b}}$ & 0.21 \\
\hline $\mathrm{XT} / \mathrm{PT}$ & $1.00 \pm 0.27^{\mathrm{a}}$ & $1.10 \pm 0.24^{\mathrm{a}}$ & $1.10 \pm 0.29^{\mathrm{a}}$ & $1.28 \pm 0.13^{\mathrm{B}}$ & 0.22 \\
\hline $\mathrm{TB}(\mu \mathrm{m})$ & $133.11 \pm 50.94^{\mathrm{A}}$ & $154.24 \pm 34.86^{\mathrm{ab}}$ & $177.84 \pm 46.39^{\mathrm{C}}$ & $181.03 \pm 37.66^{\mathrm{bc}}$ & 0.26 \\
\hline $\operatorname{TMV}(\mu \mathrm{m})$ & $394.33 \pm 60.30^{\mathrm{a}}$ & $426.38 \pm 82.82^{\mathrm{ab}}$ & $455.45 \pm 48.38^{\mathrm{B}}$ & $431.90 \pm 63.53^{\mathrm{b}}$ & 0.13 \\
\hline TRBM & $0.34 \pm 0.11^{\mathrm{a}}$ & $0.36 \pm 0.06^{\mathrm{ab}}$ & $0.38 \pm 0.08^{\mathrm{C}}$ & $0.42 \pm 0.04^{\mathrm{bc}}$ & 0.19 \\
\hline
\end{tabular}

表注同表 3 Notes see Table 3

向外部明显呈凸透镜状突出, 角质层极不发达, 牡荆 下表皮仅零星分布表皮毛, 栅栏组织厚度、海绵组织 栅栏组织厚度比、叶厚、中脉厚度等数量性状显著小 于其它群落, 这与其阴暗的光照环境相适应。相比 之下, 混交林中的告荆、酸粘叶片在许多解剖学性状 上介于灌草丛群落和侧柏林群落之间, 表现出中生/ 阳生特点。

\subsection{3 环境因子对牡荆和酸杳叶片结构的影响}

非参数相关分析表明, 郁闭度和坡度与牡荆和 酸束叶片解剖性状的相关性均不显著 (表 5、6)。总 体看来, 叶片许多性状均与多个生态因子呈显著相 关性, 表明其结构受多个生态因子综合影响。对生 态因子显著相关性状数目的排序结果来看, 土壤含 水量、空气相对湿度和风速并列第一, 说明水分和

表 5 牡荆叶片的解剖特征与生态因子之间的非参数相关关系

Table 5 Spearman correlation between leaf anatomic characters of Vitex negundo var. cannabifolia and ecological factors

\begin{tabular}{|c|c|c|c|c|c|c|c|}
\hline & Alt & MLI & SWC & $\mathrm{RH}$ & MAT & DDT & $\mathrm{WV}$ \\
\hline $\operatorname{CTUE}(\mu \mathrm{m})$ & 0.800 & 0.800 & $-1.000^{* *}$ & $-1.000^{* *}$ & 0.800 & 0.800 & $1.000^{* *}$ \\
\hline $\operatorname{TUE}(\mu \mathrm{m})$ & 0.738 & 0.738 & -0.949 & -0.949 & 0.738 & 0.738 & 0.949 \\
\hline $\operatorname{TLE}(\mu \mathrm{m})$ & -0.800 & -0.800 & 0.400 & 0.400 & -0.800 & -0.800 & -0.400 \\
\hline $\operatorname{HDLE}\left(\mathrm{mm}^{-1}\right)$ & 0.400 & 0.400 & -0.200 & -0.200 & 0.400 & 0.400 & 0.200 \\
\hline $\operatorname{SDLE}\left(\mathrm{mm}^{-2}\right)$ & 0.800 & 0.800 & $-1.000^{* *}$ & $-1.000^{* *}$ & 0.800 & 0.800 & $1.000^{* *}$ \\
\hline $\mathrm{SL}(\mu \mathrm{m})$ & 0.400 & 0.400 & -0.200 & -0.200 & 0.400 & 0.400 & 0.200 \\
\hline $\mathrm{TPT}(\mu \mathrm{m})$ & 0.800 & 0.800 & $-1.000^{* *}$ & $-1.000^{* *}$ & 0.800 & 0.800 & $1.000^{* *}$ \\
\hline $\operatorname{TST}(\mu \mathrm{m})$ & 0.400 & 0.400 & -0.800 & -0.800 & 0.400 & 0.400 & 0.800 \\
\hline TST/TPT & $-1.000^{* *}$ & $-1.000^{* *}$ & 0.800 & 0.800 & $-1.000^{* *}$ & $-1.000^{* *}$ & -0.800 \\
\hline $\mathrm{TL}(\mu \mathrm{m})$ & 0.800 & 0.800 & $-1.000^{* *}$ & $-1.000^{* *}$ & 0.800 & 0.800 & $1.000^{* *}$ \\
\hline $\mathrm{XT}(\mu \mathrm{m})$ & 0.800 & 0.800 & $-1.000^{* *}$ & $-1.000^{* *}$ & 0.800 & 0.800 & $1.000^{* *}$ \\
\hline $\mathrm{PT}(\mu \mathrm{m})$ & 0.800 & 0.800 & $-1.000^{* *}$ & $-1.000^{* *}$ & 0.800 & 0.800 & $1.000^{* *}$ \\
\hline $\mathrm{XT} / \mathrm{PT}$ & -0.800 & -0.800 & 0.600 & 0.600 & -0.800 & -0.800 & -0.600 \\
\hline $\mathrm{TB}(\mu \mathrm{m})$ & 0.800 & 0.800 & $-1.000^{* *}$ & $-1.000^{* *}$ & 0.800 & 0.800 & $1.000^{* *}$ \\
\hline $\operatorname{TMV}(\mu \mathrm{m})$ & 0.400 & 0.400 & -0.800 & -0.800 & 0.400 & 0.400 & 0.800 \\
\hline TRBM & 0.258 & 0.258 & -0.258 & -0.258 & 0.258 & 0.258 & 0.258 \\
\hline NCC & 1 & 1 & 7 & 7 & 1 & 1 & 7 \\
\hline
\end{tabular}

* : $p<0.05$ **: $p<0.01$ NCC: 与该因子显著相关的性状数目 Number of character signicantly correlated to the factors 其它见表 $1 \sim 3$ Others see Table 1,2 and 3 
表 6 酸冭叶片的解剖特征与生态因子之间的非参数相关关系

Table 6 Spearman correlation between leaf anatomic characters of Ziziphus jujuba and ecological factors

\begin{tabular}{|c|c|c|c|c|c|c|c|}
\hline & Alt & MLI & SWC & $\mathrm{RH}$ & MAT & DDT & WV \\
\hline $\operatorname{CTUE}(\mu \mathrm{m})$ & 0.800 & 0.800 & $-1.000^{* *}$ & $-1.000^{* *}$ & 0.800 & 0.800 & $1.000^{* *}$ \\
\hline $\operatorname{TUE}(\mu \mathrm{m})$ & 1.000 & $1.000^{* *}$ & -0.800 & -0.800 & $1.000^{* *}$ & $1.000^{* *}$ & 0.800 \\
\hline $\operatorname{TLE}(\mu \mathrm{m})$ & -0.200 & -0.200 & -0.400 & -0.400 & -0.200 & -0.200 & 0.400 \\
\hline $\operatorname{CTLE}(\mu \mathrm{m})$ & 0.800 & 0.800 & $-1.000^{* *}$ & $-1.000^{* *}$ & 0.800 & 0.800 & $1.000^{* *}$ \\
\hline $\operatorname{SDLE}\left(\mathrm{mm}^{-2}\right)$ & 0.800 & 0.800 & $-1.000^{* * *}$ & $-1.000^{* *}$ & 0.800 & 0.800 & $1.000^{* *}$ \\
\hline $\mathrm{SL}(\mu \mathrm{m})$ & 0.800 & 0.800 & -0.400 & -0.400 & 0.800 & 0.800 & 0.400 \\
\hline $\mathrm{TPT}(\mu \mathrm{m})$ & 0.800 & 0.800 & $-1.000^{* *}$ & $-1.000^{* *}$ & 0.800 & 0.800 & $1.000^{* *}$ \\
\hline $\operatorname{TST}(\mu \mathrm{m})$ & 0.400 & 0.400 & 0.200 & 0.200 & 0.400 & 0.400 & -0.200 \\
\hline TST/TPT & -0.800 & -0.800 & $1.000^{* *}$ & $1.000^{* *}$ & -0.800 & -0.800 & $-1.000^{* *}$ \\
\hline $\mathrm{TL}(\mu \mathrm{m})$ & 0.800 & 0.800 & $-1.000^{* *}$ & $-1.000^{* *}$ & 0.800 & 0.800 & $1.000^{* *}$ \\
\hline $\mathrm{XT}(\mu \mathrm{m})$ & 0.800 & 0.800 & $-1.000^{* * *}$ & $-1.000^{* *}$ & 0.800 & 0.800 & $1.000^{* *}$ \\
\hline $\mathrm{PT}(\mu \mathrm{m})$ & $1.000^{* *}$ & $1.000^{* *}$ & -0.800 & -0.800 & $1.000^{* *}$ & $1.000^{* *}$ & 0.800 \\
\hline $\mathrm{XT} / \mathrm{PT}$ & 0.632 & 0.632 & -0.949 & -0.949 & 0.632 & 0.632 & 0.949 \\
\hline $\mathrm{TB}(\mu \mathrm{m})$ & 0.800 & 0.800 & $-1.000^{* *}$ & $-1.000^{* *}$ & 0.800 & 0.800 & $1.000^{* *}$ \\
\hline $\operatorname{TMV}(\mu \mathrm{m})$ & $1.000^{* *}$ & $1.000^{* *}$ & -0.500 & -0.500 & $1.000^{* *}$ & $1.000^{* *}$ & 0.500 \\
\hline TRBM & 0.800 & 0.800 & $-1.000^{* *}$ & $-1.000^{* *}$ & 0.800 & 0.800 & $1.000^{* * *}$ \\
\hline NCC & 2 & 3 & 9 & 9 & 3 & 3 & 9 \\
\hline
\end{tabular}

表注同表 5 Notes see Table 5

风速是影响牡荆和酸束叶片结构的主导因子。其 中, 水分因子与叶片的上表皮角质层厚度、气孔器密 度、栅栏组织厚度、叶片厚度、中脉木质部厚度、韧皮 部厚度、维管束厚度等性状均呈显著负相关,而风速 则与上述叶片解剖性状呈显著负相关。与牡荆相 比, 酸柊叶片结构更易受生态因子的影响。

\section{4 讨论与结论}

\section{1 混交林优势树种叶片结构的特点}

由于遗传因子与环境因子的共同作用, 在旱生 植物结构的适应性上出现了两种演化趋势, 即趋同 性和趋异性(王勋陵和马䩀, 1999)。退化生态系统 的明显特征是土壤贫㾑, 生物种类稀少, 小气候的各 项指标变幅大, 淮北相山更是如此。本文研究表明, 相山混交疏林 5 个优势树种叶片都有一定的旱生特 征, 如表皮具发达的表皮毛或角质层, 全栅等面叶或 具发达的栅栏组织,维管组织发达等,这些特征是对 其干旱生境趋同适应的结果。然而, 由于不同树种 的遗传基础不同, 它们对干旱生境的适应方式又有 所不同。就表皮适应特征而言, 构树和牡荆具发达 的表皮毛,而酸杳、柘树和小叶鼠李则具有发达的角 质层, 就叶肉组织而言, 构树为全栅型, 酸束和告荆 全栅、异面混合型，而小叶鼠李、柘树则为异面叶。

4.2 广布优势树种叶片的解剖可塑性及其对恢复 演替植被异质生境的适应

尽管 Morisset 和 Boutin (1984) 曾建议将表型可 塑性分为发育可塑性和环境可塑性, 但迄今关于发
育可塑性的研究很少。史刚荣(2004)发现对 7 种常 绿阔叶植物叶片越冬叶比越夏叶更具旱生结构, 并 认为这些差异 (发育可塑性) 是常绿榈叶植物适应 冬、夏季截然不同的两种生境(生境的时间异质性) 的表现形式。本研究结果表明,混交林中牡荆和酸 束叶片结构随着季节的变化同样表现出较大的发育 可塑性, 初夏叶比中秋叶更具阳生叶的特点。从生 境条件来看, 5 月初, 树林枝叶刚刚抽出, 群落的郁 闭度较小, 而 9 月中旬树木枝叶繁茂, 郁闭度较大。 可见, 叶片结构的这种发育可塑性同样可认为是对 生境时间异质性 (群落的季相变化) 的一种适应策 略。

植物在异质环境里的进化可能导致对较宽范围 环境的普遍性适应, 也可能对异质环境的一部分特 化(Specialization)（Bazzaz，1996）。淮北相山属典型 的溶蚀残丘, 其裸露的岩石和薄㾉的土层, 营造了干 旱的生境特点, 但由于长期人工造林和封山育林, 在 局部地段形成了人工林或自然恢复演替而成的落叶 疏林, 从而形成了由草丛-灌草丛-灌丛-混交疏林 (或 人工侧柏林)的恢复演替系列, 在不同演替阶段的群 落中, 光因子和水分条件显著不同 (表 1), 从而导致 了生境的空间异质性。牡荆和酸苯作为相山的两个 广布优势种, 在适应异质生境的过程中其叶片结构 必然会发生特化。本文研究结果表明, 牡荆和酸柊 的叶片结构随植被不同恢复演替阶段表现出较大的 可塑性, 其中上、下表皮角质层厚度, 表皮毛密度、栅 栏组织厚度、海绵组织栅栏组织厚度比、叶片厚度等 
的可塑性最为显著, 说明它们更易受环境的影响。 随着群落的恢复演替, 两个广布优势种叶片厚度、上 表皮角质层厚度、表皮毛密度、栅栏组织厚度等显著 减小, 海绵组织栅栏组织厚度比明显增大。叶片结 构的变化趋势是: 灌草丛(旱生/阳生)-灌丛(旱生/ 阳生)_落叶疏林 (中生/阳生)-人工侧柏林 (中生/阴 生)。演替早期植物或先锋植物被认为具有许多阳 生性植物的特征, 演替后期或顶极群落植物则是对 荫蔽条件具有较强忍受力的类群 (赵平等, 2003)。 牡荆和酸莕叶片结构随恢复演替进程的这种变化趋 势表明, 它们不仅对恢复演替早期的干旱生境具有 较强的适应性, 而且对演替后期的荫蔽条件也具有 一定忍受力, 在植被恢复演替中具有重要作用。正 是叶片结构的这种可塑性, 为植物适应不同的群落 环境奠定了解剖学基础, 这是它们成为广布优势种 的重要机制。

关于不同演替系列中植物的可塑性一直有不同 的结论。很早就有人认为, 早期演替植物种比晚期 演替植物有更大的适应能力 ( Bazzaz, 1979)。Zangerl 和 Bazzaz (1983) 认为后期种对光和温度缺少可塑 性, 这一观点得到了 Raich (1989) 的实验支持。对温 带森林的研究表明, 中间演替树种比早期和晚期演 替树种有更大的表现型可塑性 ( Neuner \& Bannister, 1995), 中间种能够适应较宽范围的生态环境 (Abrams \& Mostoller, 1995)。本文研究的群落均处于 恢复演替的早期阶段, 生荆和酸束作为两个广布种, 其叶片结构在时间和空间尺度上均表现出明显的可 塑性, 从而支持 $\operatorname{Bazzaz}(1979)$ 的观点。

\section{3 环境因子对叶片结构的影响}

关于水分、光照和温度等生态因子对叶片结构 的影响已有不少报道(郭玉华等, 2004; 刘全宏等, 2001; 费松林等, 1999; Rôças et al., 1997; Jackson, 1967; Wylie, 1951), 但关于风速对叶片结构影响的 研究尚不多见。本文研究表明, 淮北相山牡荆和酸 隶叶片结构受诸多生态因子的综合影响, 其中水分 和风速是其主导因子。水分是相山植物生长的限制 因子, 而风速则与叶片的蒸腾作用密切相关。

已有的研究表明, 随着降水量的减少, 叶片的角 质膜增厚, 栅栏组织增厚, 层数增加, 叶片的厚度增 加(费松林等, 1999; Jackson, 1967)。淮北相山牡荆 和酸束叶片的上表皮角质层厚度、栅栏组织厚度、叶 片厚度均与土壤含水量、空气相对湿度呈显著负相 关, 与风速呈显著正相关。这一结果与已有报道相 一致, 其原因是栅栏组织层数的增加及表皮角质膜
的增厚有利于防止水分的过度蒸腾, 从而保持正常 的代谢。

气孔是植物体进行气体交换和水分蒸腾的通道 与门户, 其密度和大小主要受光照、温度和降水的影 响。随着温度的增加和水分的减少, 气孔器密度增 加, 但气孔器长宽指数则向小型化发展 (Carpenter \& Smith, 1975; Strobel \& Sundberg, 1984)。小而多的气 孔对于有效控制水分过多蒸腾具有重要作用( Weyers \& Meidner, 1990; 贺金生等, 1994)。本文研究结 果表明, 牡荆和酸疋气孔器密度均与土壤含水量、空 气相对湿度呈显著负相关, 与风速呈负相关, 但其长 度与环境因子之间的相关性不明显。可见, 环境因 子对植物气孔特征影响的大小, 因物种及物种对其 生境变化敏感性的不同而具有差异 (郑淑霞和上官 周平, 2005)。

叶脉在叶片中起着支持和输导作用, 大的中脉 可提高叶片的强度, 从而避免叶片因干旱和大风而 变形( Turner, 1994; Rôças et al., 1997)。牡荆和酸 梞中脉木质部厚度和维管束厚度等性状均与土壤含 水量、空气相对湿度呈显著负相关, 而与风速呈显著 负相关。可见, 发达中脉及其维管组织不仅有利于 提高水分的输导效率, 同时对叶片抵御干旱和大风 有着重要意义。

总之, 从相山不同演替阶段的优势种叶片解剖 结构比较分析可知, 相山 5 个优势树种叶片在恢复 演替早期阶段都表现出一定的旱生特征。相山植被 恢复过程中形成的群落环境的空间异质性及其季相 变化, 导致了广布优势种叶片的解剖可塑性, 这种可 塑性既是植物适应其异质生境的一种重要机制, 同 时又是不同群落环境, 尤其是水分条件的直接效应 的反映。

\section{参 考 文 献}

Abrams MD, Mostoller SA (1995). Gas exchange, leaf structure and nitrogen in contrasting successional tree species growing in open and under story sites during a drought. Tree Physiology, $15,361-370$.

Ashton PMS, Olander LP, Berlyn GP, Thadani R, Cameron IR (1998). Changes in leaf structure in relation to crown position and tree size of Betula papyrifera within fire-origin stands of interior cedar-hemlock. Canadian Journal of Botany, 76, 1180 1187.

Bazzaz FA (1979). The physiological ecology of plant. Annual Review of Ecology and Systematics, 10, 351-371.

Bazzaz FA (1996). Plants in Changing Environments: Linking

Physiological, Population, and Community Ecology. Cambridge 
University Press, Cambridge.

Bongers F, Popma J (1990) . Leaf characteristics of the tropical forest flora of Los Tuxtlas, Mexico. Botanical Gazette, 151, $354-$ 365 .

Cai YL (蔡永立), Da LJ (达良俊) (2002). Leaf ecological anatomy of six evergreen species of Fagaceae in the eastern subtropical area of China. Chinese Journal of Applied \& Environmental Biology (应用与环境生物学报), 8, 460 - 466. (in Chinese with English abstract)

Cai YL (蔡永立), Wang XH (王希华), Song YC (宋永昌) (1999). An ecoanatomical study on leaves of Cyclobalanopsis glauca populations in the eastern subtropical zone, China. Acta Ecologica Sinica (生态学报), 19,844-849. (in Chinese with English abstract)

Carpenter SB, Smith NB (1975). Stomatal distribution and size in southern Appalachian hard woods. Canadian Journal of Botany, $53,1153-1156$

Fang JY (方精云), Fei SL (费松林), Fan YJ (樊拥军), Cui KM (崔克明) (2000). Ecological patterns in anatomic characters of leaves and woods Fagus lucida and their climatic control in Mountain Fanjingshan, Guizhou, China. Acta Botonica Sinica (植物学报), 42, 636 - 642. (in Chinese with English abstract)

Fahn A (1964). Some anatomical adaptation of desert plant. Phytomophology, 14, 93-102.

Fahn A (1986). Structural and functional properties of trichomes of xeromorphic leaves. Annual of Botany, 57, 631- 637.

Fei SL (费松林), Fang JY (方精云), Fan YJ (樊拥军), Zhao K (赵坤), Liu XJ (刘雪皎), Cui KM (崔克明) (1999). Anational characteristics of leaves and woods Fagus lucida and their relationship to ecological factors in Mountain Fanjingshan, Guizhou, China. Acta Botonica Sinica (植物学报), 41, 498 499. (in Chinese with English abstract)

Guo YH (郭玉华), Cai ZQ (蔡志全), Cao KF (曹坤芳), Wang WL (王渭玲) (2004). Leaf photosynthetic and anatomic acclimation of four tropical rainforest tree species to different growth light conditions. Journal of Wuhan Botanical Research (武汉植物研究), 22, 240-244. (in Chinese with English abstract)

He JS (贺金生), Chen WL (陈伟烈), Wang XL (王勋陵) (1994). Morphological and anatomical features of Quercus section Suber and its adaptation to ecological environment. Acta Phytoecologica Sinica (植物生态学报), 18, 219 - 227. (in Chinese with English abstract)

Huang ZY (黄振英)，Wu H (吴鸿)，Hu ZH (胡正海) (1997)

The structures of 30 species of psammophytes and their adaptation to the sandy desert environment in Xinjiang. Acta Phytoecologica Sinica (植物生态学报), 21,521 - 530. (in Chinese with English abstract)

Jackson LWR (1967). Effect of shade on leaf structure of deciduous tree species. Ecology, 48, 498-499.
Liu QH (刘全宏), Wang XA (王孝安), Tian XH (田先华), Xiao YP (肖娅萍) (2001). Morphological and anatomical characteristics of leaf of Larix chinensis and their relationship to environmental factors in Taibaishan Mountain. Acta Botanica BorealiOccidentalia Sinica (西北植物学报), 21, 885-893. (in Chinese with English abstract)

Morisset P, Boutin C (1984). The biosystematic importance of phenotypic plasticity. In: Grant WF ed. Plant Biosystematics. Academic Press, London, 293 - 306.

Mott KA, Gibson AG, O'Leary JW (1982). The adaptive significance of amphistomatic leaves. Plant, Cell and Environment, 9, $455-460$.

Neuner G, Bannister P (1995). Frost resistance and susceptibly to ice formation during natural hardening in relation to leaf anatomy in three evergreen tree species from New Zealand. Tree Physiology, 15, $371-377$.

Peng SL (彭少麟), Li YL (李跃林)，Yu H (余华), Ren H (任海) (2002). Ecoanatomical study on leaf characteristics of dominant species in different succession stages of forest communities in Dinghushan. Journal of Tropical and Subtropical Botany (热带亚热带植物学报), 10, 1-8. (in Chinese with English abstract)

Raich JW (1989). Seasonal and spatial variation in the light environment in the tropical Dipterocarp forest and gaps. Biotropica, $21,229-302$.

Rôças G, Barros CF, Scarano FR (1997). Leaf anatomy plasticity of Alchornea triplinervia (Euphorbiaceae) under distinct light regimes in a Brazilian montane Atlantic rain forest. Trees, 11, $469-473$.

Shi GR (史刚荣) (2004). A study on ecological anatomy of leaves in 7 broad-leaved evergreen plants. Guihaia (广西植物), 24, 334 - 338. (in Chinese with English abstract)

Strauss-Debenedetti S, Bazzaz FA (1991). Plasticity and acclimation to light in tropical Moraceae of different sucessional positions. Oecologia, 87, $377-387$.

Strauss-Debenedetti S, Berlyn GP (1994). Leaf anatomical responses to light in five tropical Moraceae of different successional status. American Journal of Botany, 81, 1582 - 1591 .

Strobel DM, Sundberg MD (1984). Stomatal density in leaves of various xerophytes preliminary studies. Journal of the Minnesota Academy of Science, 49, 7-9.

Turner IM (1994). Sclerophylly: primarily protective? Functional Ecology, 8, $669-675$.

Wang WY (王为义) (1985) . A study on the structural particularity of alpine plants. Acta Biologica Plateau Sinica (高原生物学 集刊), 4, 19-32. (in Chinese with English abstract)

Wang XL (王勋陵), Ma J (马骥) (1999). A study on leaf-structure and the diversity of xerophytes ecology adaptation. Acta Ecologica Sinica (生态学报), 19, 787 - 792. (in Chinese with English abstract)

Wang XL (王勋陵), Wang J (王静) (1989). Plant Morphology 
and Environment (植物形态结构与环境). Lanzhou University Press, Lanzhou. (in Chinese)

Weyers JDB, Meidner H (1990). Methods in Stomatal Research. Longman, London.

Wylie RB (1951). Principles of foliar organization shown by sunshade leaves from ten species of deciduous dicotyledon trees. American Journal of Botany, 38, 355 - 361 .

Zangerl AR, Bazzaz FA (1983) . Responses of early and late successional species of polygonum to variations in resource availability. Oecologia, 56, $397-404$.

Zhang X (张兴), Yang XJ (杨晓杰) (2003). Study of leaves characters of 3 species and relationship between in structure and moist habitat. Journal of Science of Teacher's College and University (高师理科学刊), 23(1), 46-49. (in Chinese with English abstract)
Zhao P (赵平), Zeng XP (曾小平), Peng SL (彭少麟) (2003) . Ecological adaptation of leaf gas exchange of trees used for revegetation under different experimental light regimes. Chinese Journal of Ecology (生态学杂志), 22(3), 1-8. (in Chinese with English)

Zheng SX(郑淑霞), Shangguan ZP (上官周平)（2005）。Comparison of the leaf stomatal characteristic parameters of three plants in Loess Plateau over the last 70 years. Journal of Plant Resources and Environment (植物资源与环境学报),14(1), 1 - 5. (in Chinese with English abstract)

Zhou GT (周广泰), Liu FQ (刘风琴), Wu XM (吴学明), Fan JP (范建平) (1992). A Study on the anatomical characteristics of alpine plants in Qinghai Plateau. Journal of Qinghai Normal University (Natural Science) (青海师范大学学报 (自然科学 版) ), 6(4), 45-57. (in Chinese with English abstract)

责任编委：曹坤芳 责任编辑: 刘丽娟 